\title{
2D MIMO Radar with Coprime Arrays
}

\author{
Conghui Li \\ Department of EEE \\ Imperial College London \\ London, UK \\ Email: conghui.li15@imperial.ac.uk
}

\author{
Lu Gan \\ School of Engineering and Design \\ Brunel University \\ London, UK \\ Email: lu.gan@brunel.ac.uk
}

\author{
Cong Ling \\ Department of EEE \\ Imperial College London \\ London, UK \\ Email: cling@ieee.org
}

\begin{abstract}
In this contribution, a new class of planar coprime MIMO radar systems based on quadratic integers is proposed where the antenna locations are represented by lattice points generated by prime integers in quadratic number fields. By exploiting the coprimality of certain quadratic integers, the virtual coarrays of proposed structures enjoy a quadratic gain in parameter identifiability according to the Chinese Remainder Theorem (CRT). To avoid holes in the coarray, we present Hole-free CRT arrays with guaranteed full-rank autocorrelation matrices for subspace-based target estimation. The ring of Gaussian integers and the ring of Eisenstein integers are chosen as examples of designing coprime MIMO radar systems. It is shown that the proposed arrays achieve enhanced angular resolutions and improve the side lobe suppression. In the context of target estimation, simulations show the superior performance of the coprime MIMO structures based on CRT.
\end{abstract}

Index Terms-Coprime sensing, MIMO, sparse arrays, quadratic integers, Chinese Reminder Theorem.

\section{INTRODUCTION}

Multiple-input and multiple-output (MIMO) radar system is a novel radar technology which enjoys a broad range of applications including the direction of arrival estimation (DOA) [1], beamforming [2] and imaging [3]. An $M$-by- $N$ MIMO radar system refers to $M$ transmitting antennas and $N$ receiving antennas where each transmitting antenna radiates multiple waveforms independent from other transmitters with receiving antennas being able to receive and extract these signals by matched filters, which allows transmitting and extracting $M N$ mutually orthogonal signals because of the enlarged virtual coarray [4].

Previous studies have investigated one dimensional (1D) arrays such as generalized coprime arrays [5] and super nested arrays [6] by exploiting the concept of the virtual coarray where the spatial smoothing technology was employed on Multiple Signal Classification (MUSIC) for superior angle resolutions. In the two-dimensional (2D) array processing, lattices have been well studied wherein physical antenna subarrays can be placed on related lattices. Here the virtual coarray is naturally defined as a set of all difference vectors between lattices as an extension of the concept of 1D coarray [7]. In [8] a novel approach to further investigate the theory of the coprime array was proposed based on the Chinese remainder theorem (CRT). This paper addresses the problem of parameter identifiability and proposes a new class of array configurations, namely CRT arrays where the antenna locations are presented by lattices generated by two or more pairwisely coprime ideals in quadratic fields.
The main contribution of this paper is that it further completes the design method of coprime arrays by means of CRT and addresses the issues of geometrical configurations of CRT arrays including lattice representations of coarrays. By exploiting Eisenstein integers in the quadratic field, we put forward applications of hexagonal lattices which form the tightest packing in $2 \mathrm{D}$ and hence provide the potential to decrease the physical array aperture without sacrificing the parameter identifiability. The advantages of CRT arrays are presented in the context of MIMO radar system.

The rest of the paper is organized as follows. The fundamentals of MIMO radar are briefly reviewed in Section II. In Section III we present sparse arrays based on quadratic integers, namely CRT arrays, after which Section IV proposes a novel method which extends the spatial smoothing method for target estimation to hexagonal shape arrays. Section VI concludes the paper.

\section{FUndAMENTALS OF MIMO RADAR}

\section{A. Radiation pattern}

Let $C_{E}(\theta, \phi)$ represent the radiation pattern of one antenna element and $A F^{\prime}(\theta, \phi)$ denote the normalization of array factor $A F(\theta, \phi)$ of this antenna element:

$$
A F^{\prime}(\theta, \phi)=\left|A F(\theta, \phi) / A F(\theta, \phi)_{\max }\right|,
$$

then we can express the radiation pattern of an antenna array with identical elements by

$$
C(\theta, \phi)=C_{E}(\theta, \phi) A F^{\prime}(\theta, \phi) .
$$

A MIMO radar system consisting of $M$ transmitting antennas allocated at lattice points $\mathbf{z}_{m} \in \mathcal{Z}_{m}$ and $N$ receiving antennas allocated at $\mathbf{z}_{n} \in \mathcal{Z}_{n}$ possesses the two-way radiation pattern $C_{M N}$ which is the product of the transmitting radiation pattern and the receiving antenna pattern, i.e.,

$$
C_{M N}=C_{M}(\theta, \phi) C_{N}(\theta, \phi),
$$

where the array factors of transmitting and receiving arrays are given by

$$
A F_{M}(\theta, \phi)=\sum_{m=1}^{M} \mathbf{I}_{M, m} \exp \left(j \frac{2 \pi d}{\lambda} \mathbf{z}_{m} \sin (\theta) \cos (\phi)\right)
$$

and

$$
A F_{N}(\theta, \phi)=\sum_{n=1}^{N} \mathbf{I}_{N, n} \exp \left(j \frac{2 \pi d}{\lambda} \mathbf{z}_{n} \sin (\theta) \sin (\phi)\right)
$$


respectively, where $\mathbf{I}_{M, m}\left(\mathbf{I}_{N, n}\right)$ is determined by the weights of the $m$ th transmitter (the $n$th receiver), $d$ denotes the minimum interelement spacing and $\lambda$ represents the wavelength. From Equation (2) and (3), the two-way radiation pattern can be rewritten using the normalized array factors of transmitters and receivers:

$$
C_{M N}(\theta, \phi)=C_{E}(\theta, \phi)^{2} A F_{M}^{\prime}(\theta, \phi) A F_{N}^{\prime}(\theta, \phi) .
$$

Therefore we can consider a virtual array with normalized array factor $A F_{M N}^{\prime}(\theta, \phi)=A F_{M}^{\prime}(\theta, \phi) A F_{N}^{\prime}(\theta, \phi)$. Substituting Equation (4) and Equation (5) to $A F_{M N}(\theta, \phi)$ results

$A F_{M N}(\theta, \phi)=\sum_{m=1}^{M} \sum_{n=1}^{N} \mathbf{I}_{M, m} \mathbf{I}_{N, n} \exp \left(j \frac{2 \pi d}{\lambda} \mathbf{v}_{\theta, \phi} \mathbf{d}_{m, n}^{T}\right)$, where $\mathbf{v}_{\theta, \phi}=\sin (\theta)[\cos (\phi) \quad \sin (\theta)]$ and $\mathbf{d}_{m, n}$ is the $(m, n)$ th element in sum coarray set $\mathcal{S}=\{\mathbf{d}: \mathbf{d}=$ $\left.\mathbf{z}_{m}+\mathbf{z}_{n}\right\}$ whose cardinality limits the maximum number of targets that can be identified.

\section{B. Data Model}

Herein, the data model is similar to that in [9] where clutter is absent. Let $K$ denote the number of reflective signals of $K$ far-field uncorrelated targets which are with unit power and can be denoted as $s_{1}(t), s_{2}(t), \cdots s_{K}(t)$. The signals generated by the matched filter at the receiving antennas can be described by

$$
\mathbf{x}(t)=\sum_{k=1}^{K} \mathbf{a}_{m}\left(\theta_{k}, \phi_{k}\right) \otimes \mathbf{a}_{n}\left(\theta_{k}, \phi_{k}\right) s_{k}(t)+\mathbf{n}(t),
$$

where

$\mathbf{a}_{m}\left(\theta_{k}, \phi_{k}\right)=\exp \left(-j \frac{2 \pi d}{\lambda} \mathbf{z}_{m} \sin \left(\theta_{k}\right)\left[\cos \left(\phi_{k}\right) \sin \left(\theta_{k}\right)\right]\right)$ $\mathbf{a}_{n}\left(\theta_{k}, \phi_{k}\right)=\exp \left(-j \frac{2 \pi d}{\lambda} \mathbf{z}_{n} \sin \left(\theta_{k}\right)\left[\cos \left(\phi_{k}\right) \sin \left(\theta_{k}\right)\right]\right)$.

Assuming all targets are non-coherent, the autocorrelation matrix of received data can be expressed as

$$
\mathbf{R}_{\mathbf{x}}=E\left[\mathbf{x x}^{H}\right]=\mathbf{A} \mathbf{R}_{\mathbf{s}} \mathbf{A}^{H}+\mathbf{n} \mathbf{n}^{H},
$$

whereby we can apply the spatial smoothing technique which was described and employed in [2], [5]-[8] to estimate the target directions.

\section{CRT ARRAYS}

\section{A. CRT Array Configurations}

Definition 1 (CRT arrays): Given two coprime ideals $\mathcal{I}$ and $\mathcal{J}$ in ring $\mathcal{R}$, a CRT-based array is defined as:

$$
\mathcal{Z}=\sigma(\mathcal{I}) / \sigma(\mathcal{I} \mathcal{J}) \cup \sigma(\mathcal{J}) / \sigma(\mathcal{I} \mathcal{J})
$$

where $\sigma$ denotes the canonical embedding between algebraic integers and Euclidean space.

Definition 2 (Sum coarray of CRT arrays): The virtual coarray $\mathcal{S}$ generated by an CRT array is given by:

$\mathcal{S}=\left\{\mathbf{z}_{m}+\mathbf{z}_{n} \mid \mathbf{z}_{m} \in \sigma(\mathcal{I}) / \sigma(\mathcal{I} \mathcal{J}), \mathbf{z}_{n} \in \sigma(\mathcal{J}) / \sigma(\mathcal{I} \mathcal{J})\right\}$

In the special case when $\mathcal{I} \mathcal{J}=(p)$, expressions for the number of antennas and the parameter identifiability can be derived as follows:
Proposition 1: If $\mathcal{I} \mathcal{J}=(p)$, the total number of physical antennas is $2 p-1$ and the its parameter identifiability is $p^{2}$.

Proof: Let $\mathcal{I}=\mathfrak{p}_{1}$ and $\mathcal{J}=\mathfrak{p}_{2}$. By assumption $\mathfrak{p}_{1} \mathfrak{p}_{2}=$ $(p)$, the number of elements in $\left(\mathfrak{p}_{1}\right) / p R$ is $p$ which is the same as $\left(\mathfrak{p}_{2}\right) / p R$. Therefore the total number of elements in $\left(\mathfrak{p}_{1} / p R\right) \cup\left(\mathfrak{p}_{2} / p R\right)$ is $2 p$.

Herein, we define parameter identifiability as the maximum number of sources that can be identified which is also know as degrees of freedom [2], [3], [10]. Let $|\cdot|$ represent the cardinality of a set. According to CRT, all the sum vectors generated by coprime lattices are nonidentical, thus the total number of elements in $\left(\mathfrak{p}_{1}+\mathfrak{p}_{2}\right) / p R$ can be written as:

$$
|\mathcal{I} / p R| \cdot|\mathcal{J} / p R|=p^{2} .
$$

Note that as canonical embedding $\sigma(\cdot)$ is bijective, the number of lattice points in $\sigma(\mathcal{J}) / \sigma(\mathcal{I} \mathcal{J})$ is the same as the number of elements in $(\mathcal{J}) /(\mathcal{I} \mathcal{J})$

For example given $p=7$ in ring of Eisenstein integers, a decomposition of $p$ is $\mathfrak{p}_{1}=(2+\sqrt{3} i)$ and $\mathfrak{p}_{2}=(2-\sqrt{3} i)$ [8]. An integral basis of $\mathfrak{p}_{1}$ in $\mathbb{Z}[w]$ can be calculated by

$$
(2+\sqrt{3} i)(1, \omega)=\left(2+\sqrt{3} i, \frac{-1+3 \sqrt{3} i}{2}\right),
$$

therefore the corresponding generator matrix $\mathbf{G}_{1}$ is

$$
\begin{aligned}
\mathbf{G}_{1} & =\left(\begin{array}{cc}
2 & -\frac{1}{2} \\
\sqrt{3} & \frac{3 \sqrt{3}}{2}
\end{array}\right) \\
& =\left(\begin{array}{cc}
1 & \frac{1}{2} \\
0 & \frac{\sqrt{3}}{2}
\end{array}\right) \underbrace{\left(\begin{array}{cc}
1 & -2 \\
2 & 3
\end{array}\right)}_{\mathbf{B}_{1}} .
\end{aligned}
$$

where $\mathbf{B}_{1}$ is the matrix representation of $\sigma\left(\mathfrak{p}_{1}\right)$. Analogously, $\mathbf{G}_{2}$ can be obtained from $\mathfrak{p}_{2}$, therefore the antenna location of this array is given by the sets

$$
\begin{aligned}
\mathcal{Z}_{m}= & \left\{\mathbf{z}_{m}=\mathbf{G}_{2} \mathbf{x}_{1}, \forall \mathbf{x}_{1} \in A_{2} / \sigma(2+\sqrt{3} i)\right\}, \text { and } \\
& \mathcal{Z}_{n}=\left\{\mathbf{z}_{n}=\mathbf{G}_{1} \mathbf{x}_{2}, \forall \mathbf{x}_{2} \in A_{2} / \sigma(2-\sqrt{3} i)\right\},
\end{aligned}
$$

for transmitting and receiving antennas respectively.

\section{B. Hole-free CRT-based Arrays}

Definition 3 (Hole-free CRT array): Let $\Lambda$ be the lattice corresponding to $\mathcal{O}_{K}$ of a quadratic field $K$. Assume prime decomposition $p=\mathfrak{p}_{1} \mathfrak{p}_{2}$ in $\mathcal{O}_{K}$, with $\mathbf{G}_{1}$ and $\mathbf{G}_{2}$ their generator matrices. A Hole-free CRT array is a extended CRT-based array where $\mathbf{x}_{1} \in \Lambda \cap 2 \sigma\left(\mathfrak{p}_{1}\right)$ and $\mathbf{x}_{2} \in \Lambda \cap \sigma\left(\mathfrak{p}_{2}\right)$.

Let $\mathcal{S}_{C} \subset \mathcal{S}$ denote the set of consecutive elements in the coarray aperture. According to [8, Proposition 3], the minimum aperture of $\mathcal{S}_{C}$ is $\Lambda \cap p \Lambda$, i.e., $\Lambda \cap p \Lambda \subset \mathcal{S}_{C}$. Generally, $\mathcal{S}_{C}$ can be defined within a convex polygon, whereas in this paper, we only consider regular polygons such as square and hexagon. Next, we define the contiguous coarrays of Hole-free CRT over $\mathbb{Z}^{2}$ and $A_{2}$ respectively as examples. 


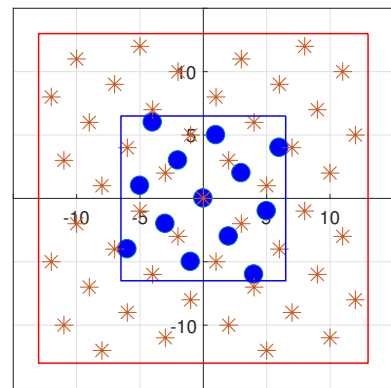

(a)

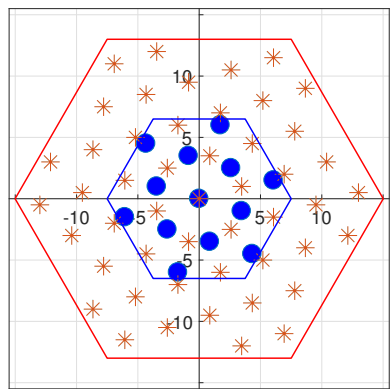

(c)

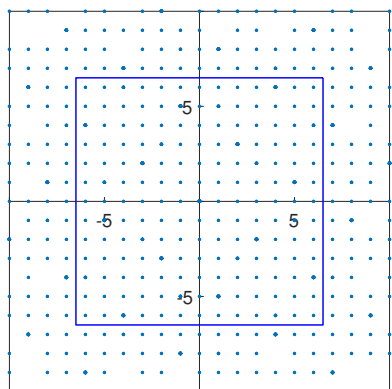

(b)

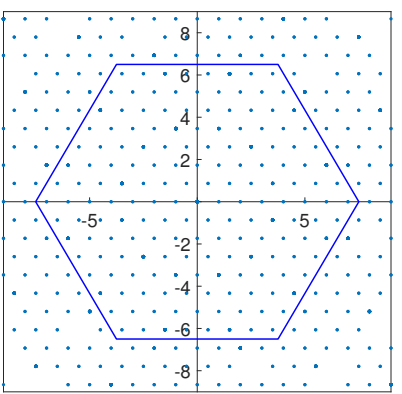

(d)
Figure 1. Hole-free CRT over $\mathbb{Z}[i]$ (a) whose contiguous sum coarray is within $13 \mathbb{Z}^{2}$ (b) and Hole-free CRT over $\mathbb{Z}[w]$ (c) whose contiguous sum coarray is within $13 A_{2}(\mathrm{~d})$. The two constituting subarrays are shown in red stars and blue dots respectively. Voronoi cells of $13 \mathbb{Z}^{2}, 26 \mathbb{Z}^{2}, 13 A_{2}$ and $26 A_{2}$ are also shown.

1) $\mathbb{Z}^{2}$ : The consecutive set of $\mathbb{Z}^{2}$ array is a uniform rectangular array (URA) which can be expressed as

$$
\begin{aligned}
\mathcal{S}_{C, G} & =\left\{\mathbf{d}=\left(d_{x, m}, d_{y, n}\right) \in \mathcal{S} \quad \mid-l_{G} \leq d_{x, m} \leq l_{G},\right. \\
-l_{G} & \left.\leq d_{y, n} \leq l_{G}, m, n=1,2, \cdots l_{G}\right\}
\end{aligned}
$$

where $l_{G} \in \mathbb{Z}$ quantizes the aperture of $\Lambda \cap p \Lambda$ and $l_{G} \geq$ $\frac{1}{2}(p-1)$. It can be calculated that the cardinality of $\mathcal{S}_{C, G}$ is $l_{G}^{2}$. Fig. 1(a) and Fig. 1(b) depict the $\mathbb{Z}^{2}$ hole free structure and its sum coarray respectively where $p=13, \mathfrak{p}_{1}=$ $(3+2 i)$ and $\mathfrak{p}_{2}=(3-2 i)$.

2) $A_{2}$ : The Hole-free part of the $A_{2}$ coarray is the hexagonal lattice whose basis is given by

$$
\mathbf{G}_{E}=\left(\begin{array}{cc}
1 & \frac{1}{2} \\
0 & \frac{\sqrt{3}}{2}
\end{array}\right) .
$$

Likewise, let $l_{r}$ denote the maximum circumradius of the contiguous hexagonal cell. According to the geometry property of hexagonal lattice, it can be calculated that $l_{r} \geq \frac{1}{2}(p+1)$. The contiguous part of the coarray of Hole-free CRT over $A_{2}$ can be described as

$$
\begin{aligned}
& \mathcal{S}_{C, E}=\left\{\mathbf{d}=\left(d_{x, m}, d_{y, m}\right) \quad \mid \mathbf{d} \in \mathcal{S},\right. \\
& -\frac{\sqrt{3}}{2} l_{r}<d_{y, m}<\frac{\sqrt{3}}{2} l_{r}, \\
& \left.-\sqrt{3} l_{r}< \pm \sqrt{3} d_{x, m}+d_{y, m}<\sqrt{3} l_{r}\right\}
\end{aligned}
$$

where $m=1,2, \cdots, 3 l_{r}^{2}+2 l_{r}+1$. An illustration of $A_{2}$ hole-free configuration and its hole-free virtual array is shown in Fig. 1(c) and Fig. 1(d) respectively where $p=$ $13, \mathfrak{p}_{1}=(1+2 \sqrt{3} i)$ and $\mathfrak{p}_{2}=(1-2 \sqrt{3} i)$.

\section{Properties of Hole-free CRT Arrays}

1) Number of Physical Antennas: According to Proposition 1 , the number of elements in $\Lambda_{1} / p R$ and $\Lambda_{2} / p R$ both equal to $p$. After doubling the range of $\mathrm{x}_{1}$ to $\mathbf{x}_{1} \in \Lambda \cap 2 \sigma\left(\mathfrak{p}_{1}\right)$ and removing the duplicated sensors at the origin, the total sensor number in $2 \Lambda_{1}$ becomes $4 p-3$. Thus the number of physical sensors by Hole-free CRT can be written as

$$
4 p-3+p=5 p-3 .
$$

2) Perimeter and Area: Assuming the minimum interelement spacing is $d$. Given a prime $p$ the perimeters of Hole-free CRT over $\mathbb{Z}^{2}$ denoted as $C_{G}$ and of Hole-free CRT over $A_{2}$ denoted as $C_{E}$ can be calculated as

$$
C_{G}=8 p d, \quad C_{E}=6 p d \tan \left(\frac{\pi}{3}\right) \approx 6.928 p d
$$

and the areas acquired by the two array configurations are $A_{G}=4 p^{2} d^{2}, A_{E}=2 p^{2} d^{2}(\tan (30)+\sec (30)) \approx 3.464 p^{2} d^{2}$.

It can be calculated that the perimeter and the area of $A_{2}$ array are $86 \%$ of those $\mathbb{Z}^{2}$ array.

\section{HEXAGON-TO-RECTANGULAR TRANSFORMATION}

In this section we transfer the hexagon shaped arrays to rectangular-like arrays by exploiting the transformation technique developed in [11]. To begin with, let us replace $\mathbf{z}_{m}$ by $\mathbf{G}_{E}\left[E_{x}, E_{y}\right]^{T}$ and rewrite $\mathbf{a}_{m}\left(\theta_{k}, \phi_{k}\right)$ as follows:

$$
\mathbf{a}_{m}\left(\theta_{k}, \phi_{k}\right)=\exp \left(-j \frac{2 \pi d}{\lambda} \mathbf{v}_{k} \mathbf{G}_{E}\left[E_{x}, E_{y}\right]^{T}\right)
$$

where $\mathbf{v}_{k}=\sin \left(\theta_{k}\right)\left[\cos \left(\phi_{k}\right) \quad \sin \left(\theta_{k}\right)\right]$. Since $\left[E_{x}, E_{y}\right]^{T}=\mathbf{G}_{E}^{-1} \mathbf{z}_{m}, E_{x}, E_{y}$ are integers with $-l_{r} \leq E_{x} \leq l_{r}$ and $-l_{r} \leq E_{y} \leq l_{r}$. The transformation from the $A_{2}$ lattice into the equivalent rectangular lattice is realized by introducing an incident wave vector in the $\mathbf{u}$ space. For every source $k$, its wave vector is defined by $\mathbf{w}_{k}=\frac{2 \pi}{\lambda} \mathbf{u}_{k}$ where $\mathbf{u}_{k}$ is expressed as

$$
\mathbf{u}_{k}=\sin \phi_{k}\left[\cos \theta_{k}, \sin \theta_{k}\right] \mathbf{G}_{E} .
$$

By substituting (18) to (17), the steering vector of the corresponding rectangular-like array on the $\mathbf{u}$ space becomes

$$
\mathbf{a}_{m}\left(\theta_{k}, \phi_{k}\right)=\exp \left(-j \frac{2 \pi d}{\lambda} \mathbf{u}_{k}\left[E_{x}, E_{y}\right]^{T}\right) .
$$

The same transformation can be done with the receiving steering vectors $\mathbf{a}_{n}\left(\theta_{k}, \phi_{k}\right)$. Here the affine transformation for an arbitrary point $\left(d_{x}, d_{y}\right) \in \mathcal{S}_{C, E}$ from Cartesian coordinate to $\mathbf{u}$ space is

$$
\left(\begin{array}{l}
E_{x} \\
E_{y}
\end{array}\right)=\left(\begin{array}{cc}
1 & -\frac{1}{\sqrt{3}} \\
0 & \frac{2}{\sqrt{3}}
\end{array}\right)\left(\begin{array}{l}
d_{x} \\
d_{y}
\end{array}\right) .
$$

In short, we transformed the CRT arrays described by the Cartesian system with $\mathbf{v}_{k}$ into an equivalent rectangular array based on the $\mathbf{u}$ space whose the wave vector is $\mathbf{w}_{k}$. After this hexagon-to-rectangular transformation, $\mathcal{S}_{C, E}$ becomes a parallelogram-like polygon in $\mathbf{u}$ space. Spatial smoothing is done using this parallelogram-like polygon array then the estimated angles are relocated back to the Euclidean space using Cartesian coordinates. 


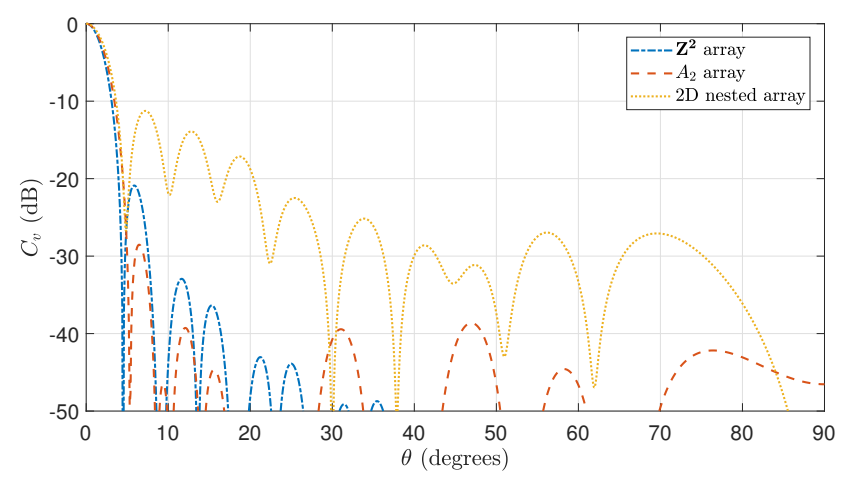

Figure 2. Radiation patterns of $\mathbb{Z}^{2}$ array, $A_{2}$ array and 2D nest array. The minimum interelement distance $d$ is chosen to be $\frac{1}{2} \lambda$. The azimuthal angle $\phi$ is set for the largest occurring side lobe.

\section{NumERICAL RESUltS}

Within the MIMO framework, the superiority of the proposed arrays is demonstrated and compared with $2 \mathrm{D}$ nested arrays in the context of the radiation pattern as well as the target estimation performance. When the decomposition parameter is selected to be $p=13$, configurations of $\mathbb{Z}^{2}$ array and $A_{2}$ array are presented in Fig. 1(a) and Fig. 1(c) respectively, whereas the array configuration of 2D nested array is given in [12] where the dense array configuration corresponding to $N_{1}=4$ acts like the transmitting array and the sparse array with $N_{2}=4$ is the receiving array.

A comparison of MIMO concepts is shown in Fig. 2. It can be measured that the side lobe suppression (SLS) of $A_{2}$ array is dramatically reduces to $\mathrm{SLS}_{A_{2}}=-25.8 \mathrm{~dB}$ which is less than SLS $_{\text {nested }}=-11.25 \mathrm{~dB}$ of the $2 \mathrm{D}$ nested array. As a result, the $A_{2}$ array configuration can target sources at a better separation. The least half power beam width is exhibited by $\mathbb{Z}^{2}$ array followed by $A_{2}$ array and finally $2 \mathrm{D}$ nested array. $\mathbb{Z}^{2}$ array improves the angular resolution by $0.8^{\circ}$ compared to the nested array. In short, all the proposed arrays outperform the known sparse array in terms of SLS and the angular resolution.

Next, the proposed arrays are employed for the target estimation on the MIMO platform. Let $K=6$ be the number of uncorrelated sources modeled as Gaussian and buried in temporally and spatially WSS noise $(\mathrm{SNR}=0$ $\mathrm{dB})$. The received data is generated by (7) and the DOA estimation is done by 2D ESPRIT technique using only 1 snapshot. From Fig. 3, it is evident that CRT arrays resolve all the sources successfully while the nested array misses one target. The root mean-square error (RMSE) is calculated by RMSE $=\sqrt{\frac{1}{K} \sum_{k=1}^{K}\left(\left(\bar{\theta}_{k}-\hat{\theta}_{k}\right)^{2}+\left(\bar{\phi}_{k}-\hat{\phi}_{k}\right)^{2}\right)}$, where $\left(\bar{\theta}_{k}, \bar{\phi}_{k}\right)$ and $\left(\hat{\theta}_{k}, \hat{\phi}_{k}\right)$ are real and estimated DOAs respectively. The results of the estimation accuracy are shown in Table I.

\section{CONCLUSiOnS}

In this paper, it has been demonstrated that the problem of designing closed-form sparse arrays can be resolved by means of quadratic integers. Inspired by the bijective mapping between the ring of algebraic integers and lattices, Chinese remaindering is performed over $\mathbb{Z}[i]$ and

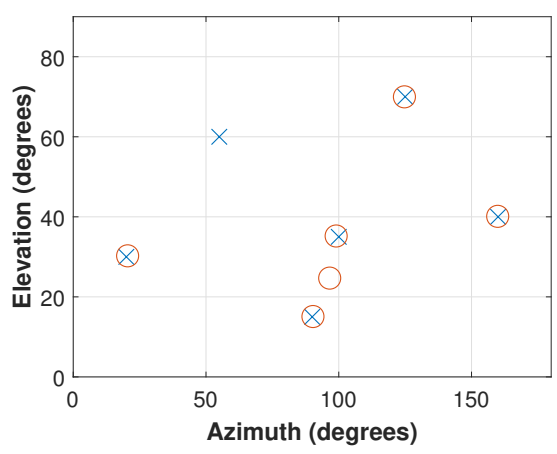

(a)

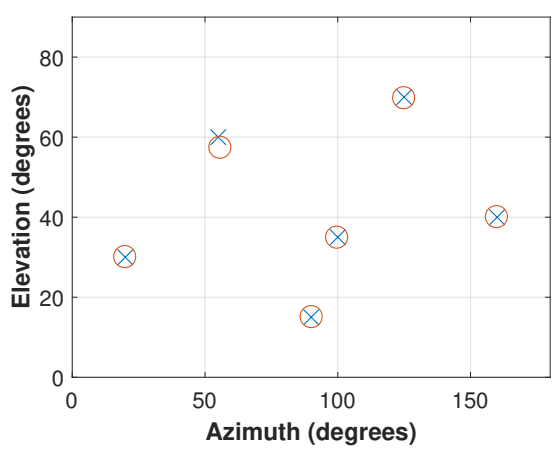

(b)

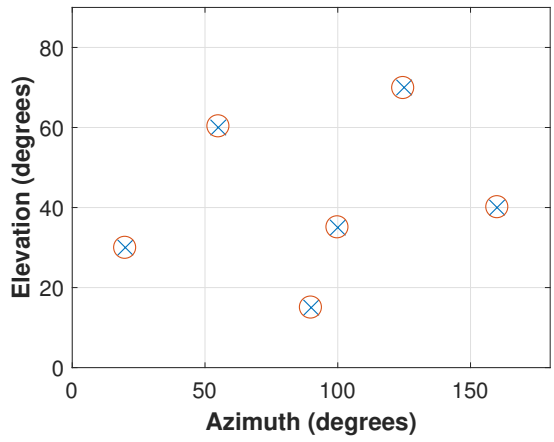

(c)

Figure 3. The true target reflectivities (in circles) and the estimated target directions (in crosses) for (a) $2 \mathrm{D}$ nested array, (b) $A_{2}$ array and (c) $\mathbb{Z}^{2}$ array

Table I

COMPARISON OF TARGET ESTIMATION

\begin{tabular}{|l||c|c|}
\hline Array & Azimuth angle & Elevation angle \\
\hline \hline $\mathbb{Z}$ array & $0.16^{\circ}$ & $0.14^{\circ}$ \\
\hline$A_{2}$ array & $0.34^{\circ}$ & $1.09^{\circ}$ \\
\hline 2D Nested array & $14.75^{\circ}$ & $8.92^{\circ}$ \\
\hline
\end{tabular}

$\mathbb{Z}[\omega]$ to generate two coprime lattices on which the transmitting and receiving arrays are allocated respectively. The proposed methodology provides a high-resolution and accuracy sensing to the allocated MIMO radar system. The superior advantages of CRT-based arrays are verified through numerical results. 


\section{REFERENCES}

[1] Y. Gu, Y. D. Zhang, and N. A. Goodman, "Optimized compressive sensing-based direction-of-arrival estimation in massive MIMO," in 2017 IEEE International Conference on Acoustics, Speech and Signal Processing (ICASSP), March 2017, pp. 3181-3185.

[2] P. Pal and P. Vaidyanathan, "Nested arrays: A novel approach to array processing with enhanced degrees of freedom," IEEE Transactions on Signal Processing, vol. 58, no. 8, pp. 4167-4181, 2010.

[3] D. Bliss and K. Forsythe, "Multiple-input multiple-output (MIMO) radar and imaging: degrees of freedom and resolution," in Signals, Systems and Computers, 2004. Conference Record of the ThirtySeventh Asilomar Conference on, vol. 1. IEEE, 2003, pp. 54-59.

[4] J. Li and P. Stoica, MIMO radar signal processing. John Wiley \& Sons, 2008.

[5] S. Qin, Y. D. Zhang, and M. G. Amin, "Generalized coprime array configurations for direction-of-arrival estimation," IEEE Transactions on Signal Processing, vol. 63, no. 6, pp. 1377-1390, March 2015.

[6] C. L. Liu and P. P. Vaidyanathan, "Super nested arrays: Linear sparse arrays with reduced mutual coupling-Part I: Fundamentals," IEEE Transactions on Signal Processing, vol. 64, no. 15, pp. 39974012, Aug 2016.

[7] P. Vaidyanathan and P. Pal, "Theory of sparse coprime sensing in multiple dimensions," IEEE Transactions on Signal Processing, vol. 59, no. 8, pp. 3592-3608, 2011.

[8] C. Li, L. Gan, and C. Ling, "Coprime sensing by chinese remaindering over rings," in Sampling Theory and Applications (SampTA), 2017 International Conference on. IEEE, 2017, pp. 561-565.

[9] S. Qin, Y. D. Zhang, and M. G. Amin, "DOA estimation of mixed coherent and uncorrelated targets exploiting coprime MIMO radar,' Digital Signal Processing, vol. 61, pp. 26-34, 2017.

[10] J. Li, P. Stoica, L. Xu, and W. Roberts, "On parameter identifiability of MIMO radar," IEEE Signal Processing Letters, vol. 14, no. 12 , pp. 968-971, 2007.

[11] Z. Tian and H. L. V. Trees, "DOA estimation with hexagonal arrays," in Acoustics, Speech and Signal Processing, 1998. Proceedings of the 1998 IEEE International Conference on, vol. 4 May 1998, pp. 2053-2056 vol.4.

[12] P. Pal and P. Vaidyanathan, "Nested arrays in two dimensions, part II: Application in two dimensional array processing," IEEE Transactions on Signal Processing, vol. 60, no. 9, pp. 4706-4718, 2012 